\title{
Combined Extracapsular Cataract Extraction and Trabeculectomy using a Separated Corneal Section
}

\author{
T. H. WILLIAMSON, A. S. BACON, D. W. FLANAGAN, C. M. JAKEMAN, \\ K. JORDAN \\ Cambridge.
}

\begin{abstract}
Summary
A retrospective study of 35 cases of extracapsular cataract extraction with posterior chamber lens implant combined with trabeculectomy was undertaken. The cataract extractions were performed through a corneal section to separate the cataract wound from the trabeculectomy. Control of intraocular pressure was achieved without medication in $77 \%$ of cases. Median improvement in visual acuity was 3.5 lines at one year. This technique provided improved trabeculectomy drainage compared with previous reports for corneoscleral incisions. Improvement in visual acuity was poorer for a group of patients with severe preoperative field loss.
\end{abstract}

The combined procedure of cataract extraction and trabeculectomy has been widely reported since the mid 1970 s. ${ }^{1,2}$ It has been shown to be effective in control of intraocular pressure and improvement of visual acuity. Recently combined extracapsular extraction, posterior chamber lens implantation and trabeculectomy has been evaluated using a corneoscleral cataract wound in continuity with the trabeculectomy flap. This has been shown reliably to improve visual acuity. However in Simmons $^{3}$ comprehensive study there was poor bleb formation at 12 months and a high incidence of postoperative pressure rises which suggest poor trabeculectomy function both in the long and short term. Fanous ${ }^{4}$ has shown that corneal sections provided improved intraocular pressure control over corneoscleral sections in a small group of patients using an intracapsular cataract extraction. Therefore the trabeculectomy flap in this study was kept separate from the cataract section by performing the latter in the peripheral cornea. The aims of this study were firstly to assess the efficacy of this technique in intraocular pressure control and improvement of visual acuity. Secondly to determine whether there was improved trabeculectomy function with a separated corneal section. Finally to see if there were any problems specific to this technique in patients with severe preoperative field loss.

\section{Materials and Methods}

Thirty-five cases of extracapsular cataract extraction with posterior chamber lens implant combined with trabeculectomy using a separated corneal section performed between 1985 and 1988 were reviewed. All patients were on treatment for glaucoma with an average intraocular pressure before the commencement of therapy of $28.6 \mathrm{mmHg}$. Eleven patients with field defects preoperatively of less than or equal to $5^{\circ}$ were classified as cases of severe glaucoma for the purposes of comparison with the group as a 
whole. Three patients who had undergone previous peripheral iridectomy or laser trabeculoplasty were included.

All patients received a standard ocular examination including field testing and fundal examination. Postoperative follow-up was at least six months and in $86 \%$ of eyes 12 months. The postoperative intraocular pressures (within three days post operation, six months and 12 months review), best visual acuity (six and 12 months), number of medications (pre and postoperative), refractive cylinder and bleb formation were noted in addition to any postoperative complications.

Surgical Procedure: After general or local anaesthesia a superior rectus suture was inserted. A fornix-based conjunctival flap was used followed by a rectangular scleral flap whilst the eye was still firm. A corneal section was performed at $1 \mathrm{~mm}$ from the limbus to allow adequate room for suturing without interference with trabeculectomy site. Twentyeight corneal sections were placed superiorly and seven temporally. The anterior capsule was excised using sodium hyaluronate to maintain the anterior chamber, the lens nucleus was removed and the soft lens material aspirated via a MacPherson two-way canula. A modified J-loop lens implant with a $5.9 \mathrm{~mm}$ u.v. filter optic was then inserted and the wound closed with $10 / 0$ nylon sutures. The trabeculectomy was then completed with sclerotomy and peripheral iridectomy. The scleral flap was sutured with $10 / 0$ nylon and the conjunctiva sutured. The procedures were carried out by four surgeons in three hospitals.

Dexamethasone $0.1 \%$, chloramphenicol $0.5 \%$ and cyclopentolate $1 \%$ drops were administered in the postoperative period.

\section{Results}

Thirty-five cases of extracapsular cataract extraction with posterior chamber lens implant combined with trabeculectomy were performed on 32 patients. Thirty-three eyes had primary open angle glaucoma and two had angle closure glaucoma. Eleven eyes had field loss preoperatively of less than or equal to $5^{\circ}$ and were classified as a group of patients with severe glaucoma for the purposes of com- parison with the group as a whole. Five patients had $15^{\circ}$ or less on field testing, four had altitudinal defects, eight had arcuate scotomas and seven were unrecordable. Median preoperative visual acuity was $6 / 36$ range from $6 / 12$ to $\mathrm{HM}$ (Table I). There was a median improvement in the visual acuity of 3.0 lines (95\% C.I. 2-4) at six months and 3.5 (95\% C.I. 2.5-4.5) at 12 months, using the Wilcoxon matched pairs signed ranks test there was a significant improvement, $\mathrm{P}<0.001$. Three patients had no improvement in visual acuity, one of these patients had disciform macular degeneration and two had severe field loss encroaching on fixation. Two patients had a reduction in visual acuity. One patient had severe preoperative field loss due to angle closure glaucoma and one dropped from hand movements to no perception of light after loss of a residual island of vision associated with a postoperative pressure rise to $28 \mathrm{mmHg}$. Patients with severe glaucomatous field defects improved significantly less as shown in Table I by MannWhitney test $\mathrm{P}<0.01$. The median improvement in visual acuity in this group was no lines at six months and only 2.0 lines at 12 months.

The mean pretherapy intraocular pressure was $28.6 \mathrm{mmHg}$ for the 35 eyes and preoperative pressure on medication $24.8 \mathrm{mmHg}$ (Table II). There was no significant difference by t-test between these figures suggesting that this group of patients did not respond to medical therapy. Improvement in the intraocular pressure was seen in 34 of the 35 patients at six months and in 28 of 30 patients at one year, the mean postoperative pressure being $9.6 \mathrm{mmHg}$ in the first three days postoperatively, $15.5 \mathrm{mmHg}$ at six months and $15.8 \mathrm{mmHg}$ at 12 months, mean drop $9.0 \mathrm{mmHg}$ at 12 months (Table III). The mean drop in the severe group was similar at $9.5 \mathrm{mmHg}$. Bleb formation was seen in all patients at six months but was lost in one patient at one year (with pressure rise from $13 \mathrm{mmHg}$ to $17 \mathrm{mmHg}$, preoperative pressure being $20 \mathrm{mmHg}$ ). One eye had a pressure rise of more then $7 \mathrm{mmHg}$ in the postoperative period. One patient had poor intraocular pressure control despite additional medical therapy at six and 12 months and one was controlled at six months but lost control at 12 
Table I Post-operative visual acuity at six months against pre-operative visual acuity for patients with combined extracapsular cataract extraction and intraocular lens implantation and trabeculectomy using a separated corneal section. Improvement in visual acuity is indicated by figures above the diagonal line. Patients with severe preoperative field loss are indicated and show a much wider scatter of improvement

\section{UISUAL ACUITY}

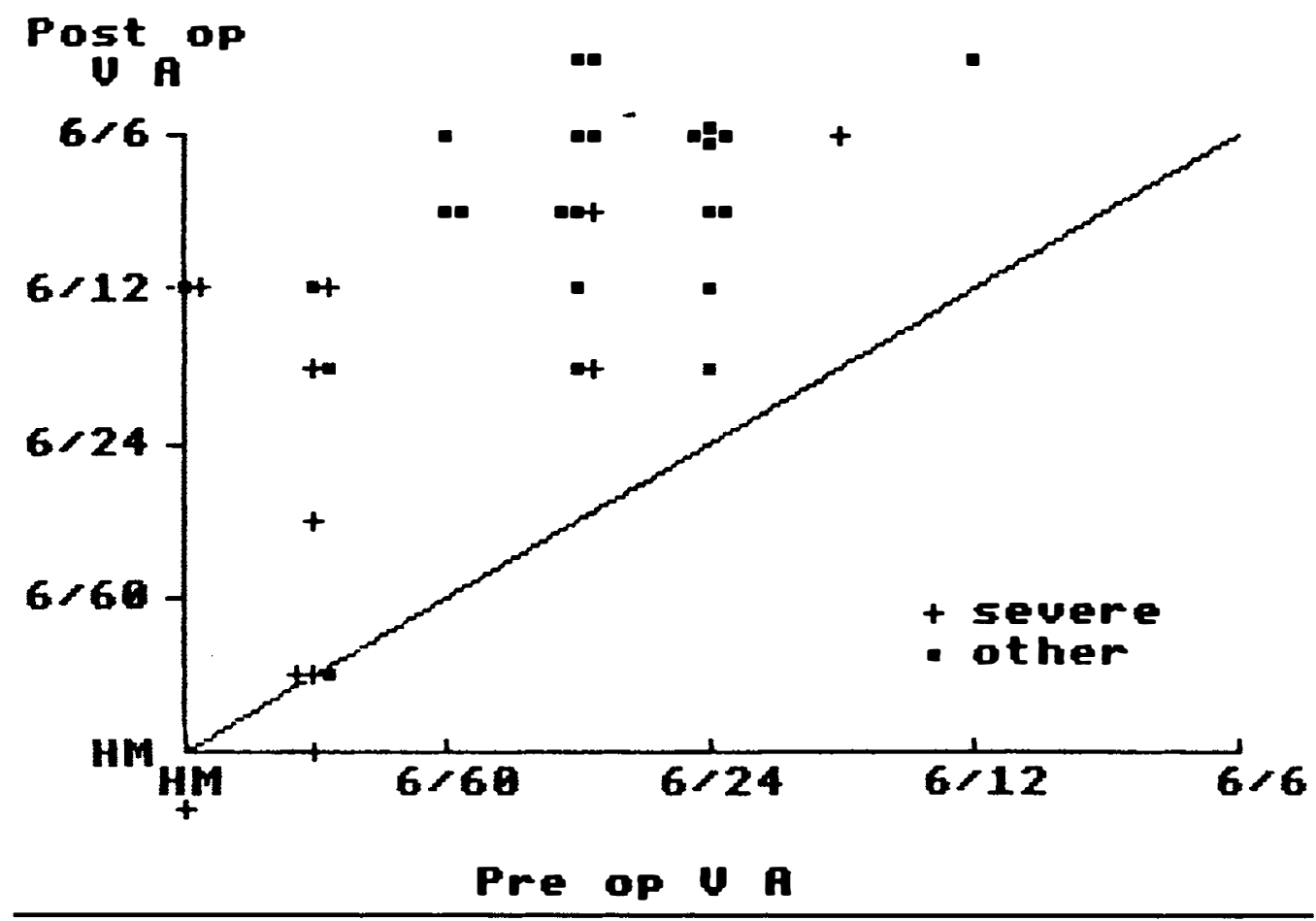

months. Both eyes had severe preoperative field loss. Postoperative medications were necessary in $23 \%$ ( $45 \%$ of the severe group) at 12 months.

Complications were encountered in 14 of the patients (Table IV). Postoperative shallowing of the anterior chamber was seen in five patients (one with wound leak not requiring surgical intervention) without lens cornea touch. This led to peripheral anterior synaechiae to the wound in two eyes but was not associated with a loss of intraocular pressure control. In five patients postoperative hyphaema occurred, and thickened posterior capsules in three, one of which was treated with YAG capsulotomy. Two patients had cystoid macular oedema, one of which had choroidal effusions in addition, the final intraocular pressure was $18 \mathrm{mmHg}$ in this case on
Timolol $0.25 \%$ and visual acuity improved from $\mathrm{HM}$ to $6 / 12$. A postoperative rise in intraocular pressure was encountered in one patient who lost residual field. There was no significant difference in the complication rates in the severe group of patients. The mean postoperative cylinder was $1.4 \mathrm{D}$, range from $-3.0 \mathrm{D}$ to $+2.0 \mathrm{D}$. The average inpatient stay was four days.

\section{Discussion}

This study shows that the combined extracapsular cataract extraction and posterior lens implantation with trabeculectomy using a separated corneal section is effective in improving visual acuity ( $63 \%$ of patients achieve $6 / 12$ vision or more) and lowering intraocular pressure. The pressure reduction is maintained over the 12 -month period in the vast majority 
Table II Intraocular pressure is shown in $\mathrm{mmHg}$ for patients with a combined extracapsular cataract extraction and trabeculectomy using a separated corneal section. A group of patients with severe pre-operative field loss is indicated. Mean pressure is shown for each period of measurement

\section{IMTRA OCULAR PRESSURES}

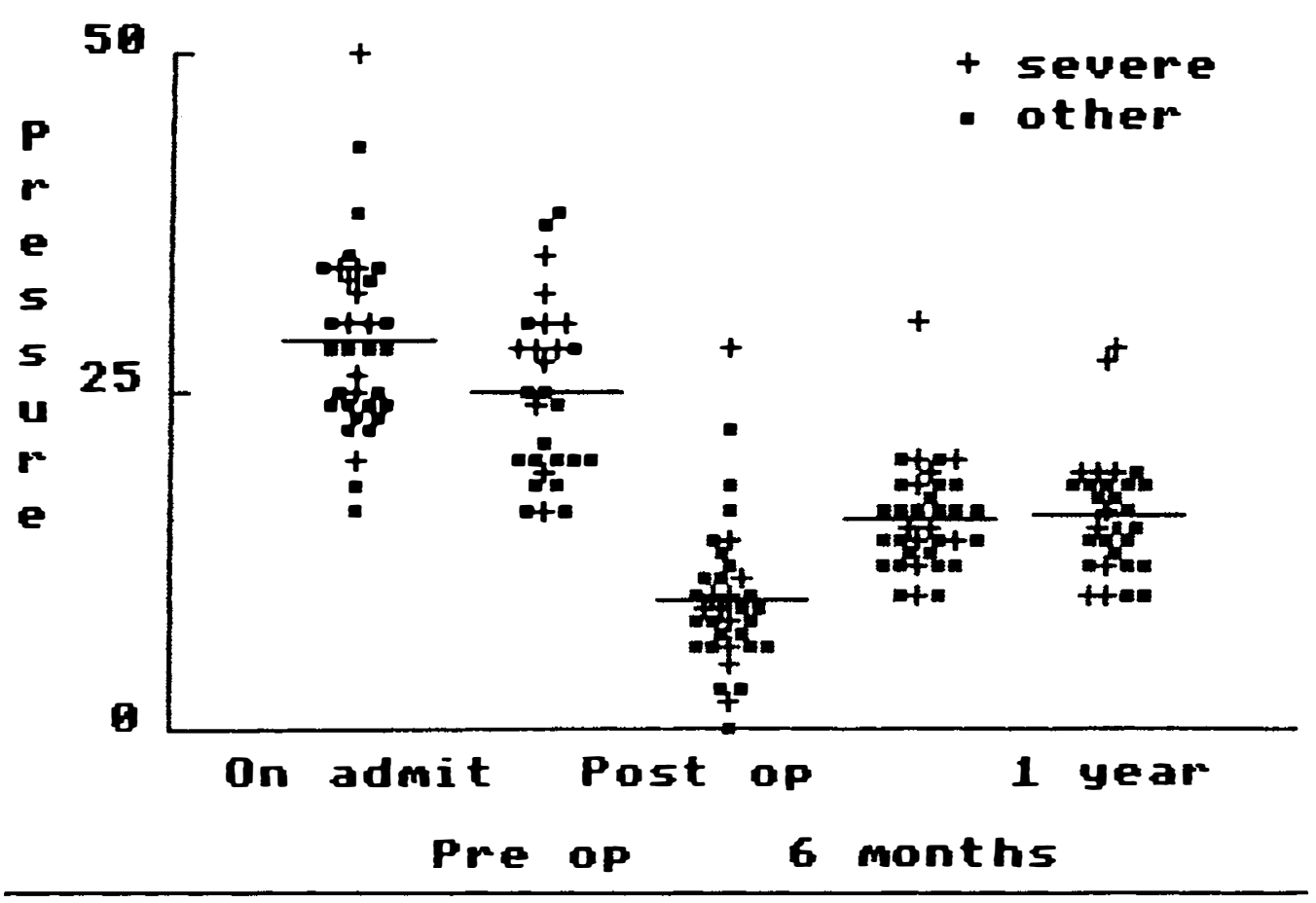

of patients, requiring further medications in only $23 \%$. The combined procedure allows both operations to be performed at one hospital admission of four days average, necessitating only one anaesthetic for this elderly group of patients.

The rate of minor complications was found to be relatively high in this study and is likely to be higher than would be encountered for trabeculectomy or cataract extraction alone. The authors did not feel, however, that there would be significantly less complications were the operations performed sequentially rather than in combined form. Indeed by performing the operations in the combined form with a separated corneal section the risk of the serious complication of postoperative pressure rises is reduced. Such pressure rises are common (23-62\%) in glaucomatous eyes after extracapsular cataract surgery alone. ${ }^{5.6}$
That they are undesirable is demonstrated in this study by the patient who lost residual field after a moderate rise in pressure to only $28 \mathrm{mmHg}$. In general however using the combined procedure with a separated corneal section, postoperative pressure rises were very uncommon $(3 \%)$. Only one patient had a rise of more than $7 \mathrm{mmHg}$.

In addition the presence of an intraocular

Table III Intraocular pressure for the combined extracapsular cataract extraction and trabeculectomy using separated corneal section

\begin{tabular}{lr}
\hline Mean & $m m \mathrm{Hg}$ \\
\hline Premedication & 28.6 \\
Preoperative & 24.8 \\
Postoperative & 9.6 \\
Six months & 15.5 \\
Twelve months & 15.8 \\
\hline
\end{tabular}


Table IV Complications of combined extracapsular cataract extraction and trabeculectomy using separated corneal section

\begin{tabular}{ll}
\hline Early & \\
Hyphaema & 5 \\
Shallow anterior chamber & 5 \\
Wound leak & 1 \\
Cystoid macular oedema & 2 \\
Choroidal effusion & 1 \\
Loss of field & 1 \\
Late & \\
Capsular thickening & 3 \\
Peripheral anterior synaechiae & 2 \\
\hline
\end{tabular}

lens may protect against lens cornea touch when shallowing of anterior chamber occurs after trabeculectomy, for although shallowing was common, occurring in five eyes in the immediate postoperative period, lens to cornea touch was not encountered. This was thought to be due to the considerably reduced bulk of the J-loop lens compared with the aged cataractous lens and not due to splinting of the anterior chamber as found with the Pearce lens?.

Despite the combination of two commonly performed procedures in one, the operation is not technically difficult to perform. This was indicated by a low peroperative complication rate of only one patient with a posterior capsule rupture which required a limited anterior vitrectomy but which still allowed implantation of a posterior chamber lens. Although corneal sections can be associated with an increased incidence of astigmatism until the sutures are removed and despite using a corneal section $1 \mathrm{~mm}$ from the limbus the postoperative refractive cylinder encountered was within an acceptable range and compares favourably with cylinders previously reported with the combined procedure using a corneoscleral section.

The method of surgery used in previous literature on combined operations has involved corneoscleral incisions as an extension of the partial thickness scleral flap $p^{3,7-10}$ or guarded sclerectomy ${ }^{11}$. These have shown good visual acuity results but evidence of poorer trabeculectomy function. In an attempt to improve trabeculectomy drainage in this study the cataract wound was kept completely separate from the trabeculectomy by performing the former through clear peripheral cornea thus avoiding any damage to the trabecular meshwork. The cataract section can be sutured without interference with the trabeculectomy site which can then be sutured loosely for improved aqueous drainage. Although differences in patient selection and methodology make direct comparisons with the previous reports in the literature using the corneoscleral section difficult some comparisons can be made in particular with Simmons trial (Table V). Loss of drainage blebs and postoperative pressure spikes were much less common in our study using a separated corneal section than in Simmons group as was the need for postoperative medications reduced. The authors felt therefore that there was some evidence for improved aqueous drainage using a separated corneal section.

The group of patients with severe preoperative field loss performed less well. There was poorer visual acuity improvement in comparison to the whole group despite a similar complication rate. Both patients who lost vision and two of the three patients who retained the same visual acuity were from this group. This poor performance was not explained by any increased incidence of concomitant eye disease and seems to be due to the severity of the glaucomatous damage present in these eyes preoperatively. Although the intraocular pressure fall was comparable with the group as a whole this required more postoperative medication. All three of the eyes with problems with pressure control belonged to this group. In addition loss of residual field is a risk when severe preoperative field loss is present.

In conclusion therefore, the combined procedure using a separated corneal section improves visual acuity and lowers intraocular pressure. There is improved trabeculectomy

Table $\mathbf{V}$ A comparison of trabeculectomy function of the separated corneal section with the continuous corneoscleral section in combined extracapsular cataract extraction and trabeculectomy

\begin{tabular}{lcc}
\hline & Separate & Continuous \\
\hline Bleb & $97 \%$ & $12 \%^{3}$ \\
Pressure spikes & $3 \%$ & $36 \%^{3}$ \\
Postoperative medications & $23 \%$ & $40-50 \% \%^{3.7}$ \\
\hline
\end{tabular}


function in comparison with studies using a trabeculectomy flap in continuity with a corneoscleral cataract section. Eyes with severe preoperative field loss improve less in regard is visual acuity and have poorer intraocular pressure control.

The authors would like to thank Judith Tees for help with the statistics.

\section{References}

${ }^{1}$ Rich W: Cataract extraction with trabeculectomy. Trans Ophthalmol Soc UK 1974, 94: 458.

2 Johns GE and Leyden WE: Combined trabeculectomy and cataract extraction. Am J Ophthalmol 1979, 88: 973-81.

${ }^{3}$ Simmons ST, Litoff D, Nichols DA, Sherwood MB, Spaeth GL: Extracapsular cataract extraction and posterior chamber intraocular lens implantation combined with trabeculectomy in patients with glaucoma. Am J Ophthalmol 1987, 104: 465-70.

${ }^{4}$ Fanous S, Brouillette G: Combined trabeculectomy and cataract extraction, a modified technique. Canad J Ophthalmol 1983, 18: 274-7.

${ }^{5}$ Savage JA, Thomas JV, Belcher CD, Simmons RJ:
Extracapsular cataract extraction and posterior chamber lens implantation in glaucomatous eyes. Ophthalmology 1985, 92: 1506-16.

${ }^{6}$ McGuigan LJB, Gottsch J, Stark WJ, Maumenee AE, Quigley HA: Extracapsular cataract extraction and posterior chamber lens implantation in eyes with prexisting glaucoma. Arch Ophthalmol 1986, 104: 1301-8.

${ }^{7}$ MacCartney DL, Memmen JE, Stark WJ et al.: The efficacy and safety of combined trabeculectomy, cataract extraction and intraocular lens implantation. Ophthalmology 1988, 95: 754-63.

8 Jay J.: Extracapsular cataract extraction and posterior chamber intraocular lens insertion combined with trabeculectomy. Br. J. Ophthalmol, 1985, 67: 487-90.

${ }^{9}$ Percival SPB: Glaucoma triple procedure of extracapsular cataract extraction, posterior chamber lens implantation and trabeculectomy. $\mathrm{Br} J$ Ophthalmol 1985, 69: 99-102.

${ }^{10}$ Neumann R, Zalish M, Oliver M: Effect of intraocular lens implantation on combined extracapsular cataract extraction with trabeculectomy: a comparative study. Br J Ophthalmol 1988, 72: 741-5.

"Shields MB: Combined cataract extraction and guarded sclerectomy. Re-evaluation in the extracapsular era. Ophthalmology 1986, 93: 366-70. 